\title{
Wie in Mathe so auch in Kunst? Zur Konzeption von Unterrichtsqualität im Kunstunterricht
}

\author{
Katrin Rakoczy (D) $\cdot$ Ernst Wagner $\cdot$ Ulrich Frick
}

Eingegangen: 15. Dezember 2020 / Überarbeitet: 30. März 2021 / Angenommen: 19. April 2021 / Online publiziert: 12. Mai 2021

(C) Der/die Autor(en) 2021

Zusammenfassung Die Frage, was guten Kunstunterricht ausmacht, stellt zwar eine der zentralen Fragen der Kunstpädagogik dar, wurde bisher jedoch kaum (quantitativ) empirisch untersucht. Im vorliegenden Beitrag werden Erkenntnisse aus der allgemeinen Unterrichtsqualitätsforschung für die Konzeption von Qualität im Kunstunterricht genutzt, indem die Dimensionen des Syntheseframeworks von Praetorius und Charalambous (2018) mit zwei zentralen kunstdidaktischen Ansätzen abgeglichen werden. Die Gegenüberstellung der aus kunstdidaktischer Sicht relevanten Unterrichtsmerkmale mit dem Syntheseframework spricht für eine grundsätzliche Eignung der aus Kernfächern bekannten Qualitätsdimensionen für den Kunstunterricht. Sie macht jedoch auch deutlich, dass eine fachspezifische Überarbeitung und Ausdifferenzierung der Dimensionen unter Berücksichtigung der Vielschichtigkeit und Komplexität des Lernprozesses notwendig ist. Es wird dargelegt, dass sich die fachspezifische Gestaltung und Ausdifferenzierung sowohl auf die Erweiterung einer Dimension (z. B. „Kognitive und emotionale Aktivierung“ statt „kognitiver Aktivierung") beziehen kann als auch auf eine unterschiedliche Schwerpunktsetzung innerhalb einer Dimension (z. B. bedeutet Klassenführung im Kunstunterricht nicht die exakte Einhaltung einer genauen Unterrichtsplanung, sondern eher die Schaffung einer kreativitätsfördernden Atmosphäre).

K. Rakoczy $(\bowtie) \cdot$ U. Frick

Hochschule Döpfer, University of Applied Sciences, Waidmarkt 3, 50676 Köln, Deutschland

E-Mail: k.rakoczy@hs-doepfer.de

U. Frick

E-Mail: u.frick@hs-doepfer.de

E. Wagner

Akademie der Bildenden Künste München, Akademiestraße 2-4, 80799 München, Deutschland

E-Mail: ernst@wagner-mchn.de 
Schlüsselwörter Unterrichtsqualität · Kunstunterricht · Ästhetische Erfahrung · Kunstpädagogik

\title{
As in Math, so in Art? On the conception of teaching quality in art instruction
}

\begin{abstract}
Although the question of what constitutes good art instruction is one of the central questions in art education, it has hardly been (quantitatively) empirically investigated so far. In this paper, insights from general teaching quality research are used to conceptualize quality in art instruction by matching the dimensions of Praetorius and Charalambous's (2018) synthesis framework with two central approaches in art education. The comparison of the instructional characteristics relevant from an art education perspective with the synthesis framework speaks for a fundamental suitability of the quality dimensions known from core subjects for art instruction. However, it also makes clear that a subject-specific revision and differentiation of the dimensions is necessary, taking into account the multi-layeredness and complexity of the learning process in art instruction. It is shown that subject-specific design can refer both to the expansion of a dimension (e.g., "cognitive and emotional activation" instead of "cognitive activation") and to a different emphasis within a dimension (e.g., classroom management in art instruction does not mean exact adherence to precise lesson planning, but rather the creation of an atmosphere conducive to creativity).
\end{abstract}

Keywords Teaching quality $\cdot$ Art instruction $\cdot$ Aesthetic experience $\cdot$ Art education

\section{Gegenstand und Lernziele im Kunstunterricht}

Obgleich die Frage, was guten Kunstunterricht ausmacht, eine der zentralen Fragen der Kunstpädagogik ist, wurde sie bisher kaum (quantitativ) empirisch untersucht. Empirische Untersuchungen in der allgemeinen Unterrichtsqualitätsforschung konzentrierten sich vielmehr auf schulische Kernfächer, insbesondere Mathematik, und ließen Fächer wie Kunst weitgehend außen vor. Die geringe Beachtung von Kunstunterricht in empirischen Studien liegt vermutlich zum einen an der mangelnden Aufmerksamkeit und der damit verbundenen geringen Zuweisung von Ressourcen gegenüber diesem „Nebenfach“ (z. B. Rittelmeyer 2013). Zum anderen erschwert die implizite Widersprüchlichkeit des Faches, also die Tatsache, dass häufig nicht eindeutig und intersubjektiv festgelegt werden kann, was richtig und was falsch bzw. was gut und was schlecht ist, seine empirische Untersuchung anhand der in der allgemeinen Unterrichtsqualitätsforschung entwickelten Kriterien; nach Ansicht einiger Fachdidaktiker*innen ist sie sogar unvereinbar mit der in Kernfächern etablierten Kompetenzorientierung (vgl. z. B. Parmentier 2011; Krautz 2007). Gegenstand des Kunstunterrichts ist z. B. eine Kunst, in der Dürers altmeisterliche Feinmalerei ebenso zum maßstabsetzenden Meisterwerk erklärt wird wie Duchamps „Urinoir“ oder Malewitschs „Weißes Quadrat auf Weißem Grund“. Entsprechend werden im Kunstunterricht nicht nur die in Kernfächern angestrebten und selbstverständ- 
lich mehrdimensionalen Bildungswirkungen bei Lernenden (z. B. Vieluf et al. 2020) gefördert; es soll darüber hinaus zumindest ein Möglichkeitsraum für sinnlich-ästhetische Wahrnehmung, Erfahrung und Erleben entstehen (z.B. Schmidt 2016) so der Konsens in der Fachdidaktik. Es sind die ästhetischen Erfahrungen, die als zentral angesehen werden, weil sie die Auseinandersetzung der Lernenden mit dem eigenen Ich in Bezug zur Welt unterstützen (Otto 1994) und damit zum für den Kunstunterricht besonders relevanten Ziel der Selbstentfaltung und Selbstwirksamkeitserfahrung beitragen können (Kirchner und Kirschenmann 2015). Lernen im Kunstunterricht wird damit verstanden als ,ein leib-sinnliches, reflexives Lernen in einer rezeptiven, gestalterischen, bildnerischen oder gar künstlerischen Auseinandersetzung bezogen auf die Wahrnehmungs-, Vorstellungs-, Darstellungs- und Mitteilungsfähigkeit“ (Berner 2016, S. 2; vgl. auch Krautz 2015). Die Frage, welche fachlichen Kompetenzen im Kunstunterricht zu erzielen sind, ist immer wieder Gegenstand intensiver Diskussionen (Peez 2013; Wagner 2018). Um die kunstpädagogische Grundintention des Ermöglichens ästhetischer Erfahrungen im Bildnerischen mit einer zunehmenden Kompetenzorientierung im Bildungswesen $\mathrm{zu}$ verbinden, wurde das Konzept der „Bildkompetenz“ entwickelt (Wagner und Schönau 2016). Es sieht die Stärkung spezifischer produktiver bildnerisch-ästhetischer und visueller Teilkompetenzen ebenso vor wie das ästhetische Erleben künstlerischer Inhalte.

\section{Kunstdidaktik und allgemeine Unterrichtsqualitätsforschung - zwei Perspektiven auf Unterrichtsqualität im Kunstunterricht}

Wie kann der komplexe und vielschichtige Lernprozess im Kunstunterricht durch didaktisches Handeln angeregt werden? Dies soll in der vorliegenden Stellungnahme anhand zweier zentraler (deutschsprachiger) kunstdidaktischer Quellen und ihren Bezügen zum Syntheseframework herausgearbeitet werden ${ }^{1}$. Zum einen wird die Konzeption von Berner (2016) herangezogen, da sie acht für das Fach Kunst formulierte Qualitätsdimensionen beschreibt, die ihrerseits ausgehend von den drei allgemeinen Qualitätsdimensionen Kognitive Aktivierung, Klassenführung und Unterrichtsklima (Klieme und Rakoczy 2008) unter Einbezug von in der einschlägigen Literatur relevanten Unterrichtsmerkmalen (zusammenfassend Hattie 2009; Helmke 2015; Meyer 2004) entwickelt wurden. Zum anderen gelten die von Kirchner und Kirschenmann (2015) beschriebenen Elemente didaktischen Handelns aufgrund ihrer weiten Verbreitung und breiten Akzeptanz innerhalb der Kunstdidaktik als zentrale Quelle. Sie wurden zwar nicht als analytische Dimensionen formuliert und auch nicht empirisch überprüft, bilden aber im Kern ähnliche Anforderungen an qualitätsvollen Kunstunterricht ab wie die fachdidaktischen Dimensionen von Berner (2016). In Tab. 1 werden sie den entsprechenden Dimensionen von Berner zugeordnet. Dabei werden Gemeinsamkeiten, aber auch Unterschiede innerhalb der Kunstdidaktik ersichtlich, z. B. in Bezug auf das Üben. Die von Berner (2016) herausgestellte Be-

\footnotetext{
1 Auch in einigen wenigen internationalen Studien (z. B. Seidel et al. 2009) wird die Qualität von Kunstunterricht empirisch untersucht. Allerdings bleiben umfassende Konzeptionen von Unterrichtsqualität hier aus, so dass sich zunächst auf die beiden zentralen deutschsprachigen Quellen bezogen wird.
} 
Tab. 1 Gegenüberstellung zweier kunstdidaktischer Ansätze und dem Syntheseframework

\begin{tabular}{|c|c|c|}
\hline $\begin{array}{l}\text { Fachdidaktisch relevante Dimensionen } \\
\text { Berner (2016) }\end{array}$ & Kirchner und Kirschenmann (2015) & $\begin{array}{l}\text { Syntheseframework } \\
\text { (Praetorius und } \\
\text { Charalambous 2018) }\end{array}$ \\
\hline $\begin{array}{l}\text { Unterricht läuft gut organisiert (1) } \\
\mathrm{ab}\end{array}$ & $\begin{array}{l}\text { Organisation von Material, Werk- } \\
\text { zeugen, Raum } \\
\text { Vielfalt der eingesetzten bildneri- } \\
\text { schen Techniken }\end{array}$ & Klassenführung \\
\hline $\begin{array}{l}\text { Denkprozesse werden initiiert (2) } \\
\text { und Erfahren und Erleben ermög- } \\
\text { licht (3) }\end{array}$ & $\begin{array}{l}\text { „Gute Aufgaben“, die nicht nur } \\
\text { kognitiv zu lösen sind }\end{array}$ & $\begin{array}{l}\text { Kognitive und emotio- } \\
\text { nale Aktivierung }\end{array}$ \\
\hline $\begin{array}{l}\text { Kreativitätsförderndes } \\
\text { Unterrichtsklima (4) }\end{array}$ & $\begin{array}{l}\text { Erfahrungsoffene Lernsettings und } \\
\text { Atmosphäre } \\
\text { Unerwartete Ergebnisse werden } \\
\text { zugelassen } \\
\text { Anwendung verschiedener Arbeits- } \\
\text { formen (Abwechslung) }\end{array}$ & $\begin{array}{l}\text { Sozioemotionale } \\
\text { Unterstützung/ } \\
\text { kreativitätsförderndes } \\
\text { Klima }^{\text {a }}\end{array}$ \\
\hline $\begin{array}{l}\text { Lernprozesse werden sichtbar } \\
\text { gemacht und -ergebnisse } \\
\text { rückgemeldet (5) }\end{array}$ & $\begin{array}{l}\text { Feedback, auch Peer- \& Selfassess- } \\
\text { ment } \\
\text { Transparente Bewertung }\end{array}$ & Formatives Assessment \\
\hline $\begin{array}{l}\text { Unterricht läuft klar verständlich ab } \\
\text { (,,intendiertes } \\
\text { Unterrichtshandeln“, 6) }\end{array}$ & $\begin{array}{l}\text { Strukturierung des Unterrichtspro- } \\
\text { zesses } \\
\text { Auswahl des Gegenstandes }\end{array}$ & $\begin{array}{l}\text { Auswahl und } \\
\text { Thematisierung des } \\
\text { Inhalts }\end{array}$ \\
\hline $\begin{array}{l}\text { Ausrichtung des Unterrichts an den } \\
\text { Lernenden und ihren Interessen (7) }\end{array}$ & $\begin{array}{l}\text { Beteiligung und Partizipation } \\
\text { Alle Schüler*innen unterstützen } \\
\text { Differenzierung } \\
\text { Individuelle Förderung }\end{array}$ & $\begin{array}{l}\text { Unterstützung des } \\
\text { Lernens aller } \\
\text { Schüler*innen }\end{array}$ \\
\hline $\begin{array}{l}\text { Ermöglichen von bewusstem } \\
\text { Üben (8) }\end{array}$ & $\begin{array}{l}\text { Geringe Bedeutung von Üben (nur } \\
\text { gezielt, z. B. für „Abzeichnen“) }\end{array}$ & Üben \\
\hline
\end{tabular}

${ }^{a}$ Es wird eine veränderte Bezeichnung der Dimensionen für den Kunstunterricht vorgeschlagen, um deutlich zu machen, dass das Verständnis der Konstrukte über die im Syntheseframework vorgesehenen hinausgeht

deutung bewussten Übens wird von Kirchner und Kirschenmann (2015) nicht geteilt. Üben findet sich demnach nicht im konsensfähigen kunstpädagogischen Diskurs wieder, der sich eher am unmittelbaren, spontanen, unverbildeten schöpferischen Ausdruck vom Kleinkind bis zum professionellen Künstler orientiert (Kirchner und Kirschenmann 2015). Darüber hinaus wird auch deutlich, dass sozio-emotionale Unterstützung in der Konzeption von Kirchner und Kirschenmann (2015) nicht explizit mitgedacht ist. Um die Passung zwischen den beiden kunstdidaktischen Positionen mit dem Syntheseframework zu beurteilen, werden in Tab. 1 die von Berner (2016) bzw. Kirchner und Kirschenmann (2015) angenommenen fachdidaktischen Qualitätskriterien und die sieben Aspekte des Syntheseframeworks gegenübergestellt.

Die Gegenüberstellung der aus kunstdidaktischer Sicht relevanten Unterrichtsmerkmale mit dem in der allgemeinen Unterrichtsqualitätsforschung mit Fokus auf Mathematik entwickelten Syntheseframework von Praetorius und Charalambous (2018) zeigt, dass die im Framework vorgenommenen Erweiterungen des im deutschsprachigen Raum häufig zugrunde gelegten Modells der drei Basisdimensio- 
nen (Praetorius et al. 2018) auch für den Kunstunterricht prinzipiell passend, jedoch nicht ausreichend sind. Trotz großer Überlappung werden Unterschiede deutlich und eine gezielte Schwerpunktsetzung und inhaltliche Spezifizierung der Unterrichtsmerkmale für den Kunstunterricht ist weiterhin erforderlich. Beispielsweise machen die beiden kunstdidaktischen Quellen deutlich, dass kognitive Aktivierung, wie wir sie aus der Mathematik kennen, dem Unterrichtsgegenstand nicht gerecht wird bzw. alleine nicht ausreicht, um eine vertiefte Auseinandersetzung mit dem Lerngegenstand zu erreichen. Kunstwerke vereinigen sinnliche, emotionale und rationale Aspekte und ihre Entschlüsselung kann nicht auf die kognitive Ebene beschränkt sein (Fuchs 2011). Deswegen spielt die Ermöglichung von (ästhetischen) Erfahrungen und von Erleben durch entsprechende Aufgaben hier eine zentrale Rolle (vgl. Schmidt 2016). Entsprechend wird vorgeschlagen, die Dimension als ,kognitive und emotionale Aktivierung“ zu bezeichnen (vgl. Schmidt 2016). Ähnliches gilt für formatives Assessment, das im Kunstunterricht weniger auf die erzielte Leistung (z. B. die Qualität der von den Schüler*innen angefertigten Artefakte) abhebt, sondern durch vertiefte Reflexionsphasen das Nachdenken, (ästhetische) Urteilen und Sprechen über eigene und fremde Arbeiten unterstützen soll (Peez 2013). Auch zeigt sich bei genauerem Hinsehen, dass die Dimensionen der Kunstdidaktik und des Syntheseframeworks sehr unterschiedlich breit definiert sind. Kreativitätsförderndes Klima bspw. ist deutlich umfassender zu verstehen als sozio-emotionale Unterstützung und weist damit auch Überlappungen zur kognitiven Aktivierung, zur Auswahl und Thematisierung des Inhalts und zur Unterstützung aller Lernenden auf (vgl. Theurer 2014). Entsprechend wird vorgeschlagen, die Dimension der sozioemotionalen Unterstützung für den Kunstunterricht zu erweitern und als kreativitätsförderndes Klima zu bezeichnen. Schließlich kann als Anmerkung zum Framework (nicht nur) in Bezug auf Kunstunterricht festgehalten werden, dass die Dimensionen nicht als trennscharf zu betrachten sind. So ist die Dimension ,Unterstützung des Lernens aller Schüler*innen“ als Anforderung konzipiert, die quer zu den übrigen Dimensionen liegt, da sie durch diese umgesetzt werden soll (z.B. durch Aufgaben mit unterschiedlichem Anforderungsniveau im Bereich der kognitiven Aktivierung oder durch das Thematisieren fachlicher Inhalte, die sich auf verschiedene Lebenswelten der Lernenden beziehen etc.).

\section{Eigene Positionierung zur Frage der Fachspezifität von Unterrichtsqualität}

Die empirische Prüfung eines Teils der im Syntheseframeworks angenommenen Qualitätsdimensionen für den Kunstunterricht in der Sekundarstufe wurde von Rakoczy et al. (im Druck) erstmalig vorgenommen und spricht für eine grundsätzliche Eignung der aus Kernfächern bekannten Qualitätsdimensionen. Die Ergebnisse unterstreichen jedoch die oben bereits ausgeführte Forderung nach fachspezifischer Überarbeitung und Ausdifferenzierung der Dimensionen unter Berücksichtigung der Vielschichtigkeit und Komplexität des Lernprozesses in Kunst in unterschiedlichen Altersgruppen und der Besonderheiten des Lerngegenstands. Wie eine solche fachspezifische Formulierung aussehen kann, wird im Folgenden für die Dimension der 
Klassenführung beispielhaft dargestellt: Vor allem ästhetische Prozesse benötigen andere zeitliche Rhythmen und v. a. Freiraum. Offenheit und Komplexität gelten daher als wichtige Bestandteile des Kunstunterrichts. Anweisungen werden deshalb oft bewusst so formuliert, dass sie in komplexe, zunächst unklare und offene Prozesse führen (Berner 2016), die dann darüber hinaus stark individualisiert sind. Der Schwerpunkt der Klassenführung liegt somit nicht auf der exakten Einhaltung einer genauen Unterrichtsplanung, sondern eher auf der Schaffung einer kreativitätsfördernden (Werkstatt-/Studio-/Atelier-)Atmosphäre, die eine Durchführung von Unterricht in flexiblerer Weise notwendig macht.

Aufbauend auf die durch Verankerung in der kunstdidaktischen Literatur fachspezifisch formulierten Dimensionen sollten spezifische Messinstrumente entwickelt werden, um ein möglichst präzises Bild der einzelnen Merkmale und Prozesse der Unterrichtsqualität zeichnen zu können. Dadurch können weiterführende Erkenntnisse über die Ausgestaltung und Wirksamkeit von Kunstunterricht gewonnen werden, die zur Verbesserung von Kunstunterricht beitragen können (erste Beispiele für solche Studien: Rakoczy et al., im Druck angenommen; Orth 2018; Schmidt 2016; Theurer 2014).

Generell ist bei der Konzeption und Untersuchung von Unterrichtsqualität im Kunstunterricht neben den oben behandelten Input- und Prozesskriterien ein besonderes Qualitätsmerkmal zu berücksichtigen: Im Kunstunterricht entsteht im kreativen Prozess ein geschaffenes Produkt, das für die Lernenden eine komplexe Symbolisierung eines je spezifischen Selbst- und Weltverhältnisses darstellt. Die damit verbundenen Erfahrungen gehen - im gelungenen Fall - von Selbstwirksamkeit bis zu produktiver Fremdheit. Wir müssen also für dieses Fach ein klassisches Output-Kriterium (z. B. Bildkompetenz) konzipieren und erfassen, dessen Rückwirkung auf die Prozessqualitäten nicht zu unterschätzen ist (Kirchner und Kirschenmann 2015) und das die Anschlussfähigkeit kunstdidaktischer Unterrichtsqualitätsforschung erhöht. Der Charakter des geschaffenen Produkts unterscheidet das Fach nicht nur deutlich von den „Kernfächern“, sondern auch von den künstlerischen Fächern, deren performativen Produkte (z.B. Konzert, Theateraufführung) flüchtig sind.

Funding Der Text ist im Rahmen des Verbundprojekts „Bildkompetenz in der Kulturellen Bildung“ entstanden, welches vom Bildunsministerium für Bildung und Forschung unter den Kennzeichen 01JK1606A und 01JK1606B gefördert wird.

Funding Open Access funding enabled and organized by Projekt DEAL.

Open Access Dieser Artikel wird unter der Creative Commons Namensnennung 4.0 International Lizenz veröffentlicht, welche die Nutzung, Vervielfältigung, Bearbeitung, Verbreitung und Wiedergabe in jeglichem Medium und Format erlaubt, sofern Sie den/die ursprünglichen Autor(en) und die Quelle ordnungsgemäß nennen, einen Link zur Creative Commons Lizenz beifügen und angeben, ob Änderungen vorgenommen wurden.

Die in diesem Artikel enthaltenen Bilder und sonstiges Drittmaterial unterliegen ebenfalls der genannten Creative Commons Lizenz, sofern sich aus der Abbildungslegende nichts anderes ergibt. Sofern das betreffende Material nicht unter der genannten Creative Commons Lizenz steht und die betreffende Handlung nicht nach gesetzlichen Vorschriften erlaubt ist, ist für die oben aufgeführten Weiterverwendungen des Materials die Einwilligung des jeweiligen Rechteinhabers einzuholen.

Weitere Details zur Lizenz entnehmen Sie bitte der Lizenzinformation auf http://creativecommons.org/ licenses/by/4.0/deed.de. 


\section{Literatur}

Berner, N. (2016). Unterrichtsqualität im Fach Kunst. Kunst + Unterricht EXKURS Beilage Heft 407/408. Friedrich.

Fuchs, M. (2011). Ästhetisches Lernen und Lehren in Institutionen. Diskurse- Themen - Zugänge. In Akademie Remscheid (Hrsg.), Jahrbuch Kulturpädagogik der Akademie Remscheid. Remscheid: Akademie Remscheid.

Hattie, J. (2009). Visible learning. A synthesis of over 800 meta-analyses relating to achievement. New York, NY: Routledge.

Helmke, A. (2015). Unterrichtsqualität und Lehrerprofessionalität: Diagnose. Evaluation und Verbesserung des Unterrichts. Seelze: Klett, Kallmeyer.

Kirchner, C., \& Kirschenmann, J. (2015). Kunst unterrichten. Didaktische Grundlagen und schülerorientierte Vermittlung. Seelze: Klett, Kallmeyer.

Klieme, E., \& Rakoczy, K. (2008). Empirische Unterrichtsforschung und Fachdidaktik. Outcome-orientierte Messung und Prozessqualität des Unterrichts. Zeitschrift für Pädagogik, 54, 222-237.

Krautz, J. (2007). Ware Bildung - Schule und Universität unter dem Diktat der Ökonomie. : Diederichs.

Krautz, J. (2015). Ich, wir. Welt. Zur Systematik und Didaktik einer personalen Kunstpädagogik. In A. Glas, U. Heinen, J. Krautz, M. Miller, H. Sowa \& B. Uhlig (Hrsg.), Kunstunterricht verstehen. Schritte zu einer systematischen Theorie und Didaktik der Kunstpädagogik (S. 13-26). : kopaed.

Meyer, H. (2004). Was ist guter Unterricht? Berlin: Cornelsen

Orth, S. (2018). Über plastische Schülerarbeiten sprechen. Eine empirische Studie zu Reflexionsphasen im Kunstunterricht der Grundschule. Kopaed.

Otto, G. (1994). Das Ästhetische ist „Das Andere der Vernunft“. Der Lernbereich Ästhetische Erziehung. Friedrich Jahresheft XII. (S. 56). Seelze: Klett, Kallmeyer.

Parmentier, M. (2011). Ästhetische Lust. In Kunstportal. Schroedel.

Peez, G. (2013). Kunstpädagogik. In Kulturelle Bildung. https://doi.org/10.25529/92552.294. https://www. kubi-online.de/artikel/kunstpaedagogik.

Praetorius, A.-K., \& Charalambous, C. Y. (2018). Classroom observation frameworks for studying instructional quality: looking back and looking forward. ZDM Mathematics Education, 50(3), 535-553.

Praetorius, A.-K., Klieme, E., Herbert, B., \& Pinger, P. (2018). Generic dimensions of teaching quality: the German framework of three basic dimensions. ZDM Mathematics Education, 50, 407-426. https:// doi.org/10.1007/s11858-018-0918-4.

Rakoczy, K., Frick, U., Weiß, S., Tallon, M., \& Wagner, E. (im Druck) Unterrichtsqualität im Kunstunterricht - wie wird sie von Lernenden wahrgenommen und wie hängt sie mit ihrer Motivation zusammen? In A. Scheunpflug, S. Timm \& I. Züchner (Hrsg.), Sonderheft der Zeitschrift für Erziehungswissenschaft zum Thema „Kulturelle Bildung “ (angenommen).

Rittelmeyer, C. (2013). Die Erforschung von Transferwirkungen künstlerischer Tätigkeiten. In Kulturelle Bildung online. https://doi.org/10.25529/92552.282. https://www.kubi-online.de/artikel/erforschungtransferwirkungen-kuenstlerischer-taetigkeiten.

Schmidt, R. (2016). Mit Kunstwerken zum Denken anregen. Eine empirische Untersuchung zur kognitiven Aktivierung im Rahmen der Kunstrezeption der Grundschule. Kopaed.

Seidel, S., Tishman, S., Winner, E., Hetland, L., \& Palmer, P. (2009). The qualities of qualitiy. Understanding excellence in arts education. Cambridge: Harvard University. https://www.wallacefoundation.org/ knowledge-center/Documents/Understanding-Excellence-in-Arts-Education.pdf

Theurer, C. (2014). Kreativitätsförderndes Klassenklima als Determinante der Kreativitätsentwick-lung von Grundschulkindern. Dissertation. Kassel: Universität Kassel.

Vieluf, S., Praetorius, A.-P., Rakoczy, K., Kleinknecht, M., \& Pietsch, M. (2020). Angebots-NutzungsModelle der Wirkweise des Unterrichts: eine kritische Auseinandersetzung mit ihrer theoretischen Konzeption. In E. Klieme, A.-P. Praetorius \& J. Grünkorn (Hrsg.), 66. Beiheft der Zeitschrift für Pädagogik: Empirische Forschung zu Unterrichtsqualität. Theoretische Grundfragen und quantitative Modellierungen (S. 63-80).

Wagner, E. (2018). Bildkompetenz - Visual Literacy. Kunstpädagogische Theorie- und Lehrplanentwicklungen im deutschen und europäischen Diskurs. In: Kulturelle Bildung online. https://www.kubionline.de/artikel/bildkompetenz-visual-literacy-kunstpaedagogische-theorie-lehrplanentwicklungendeutschen https://doi.org/10.25529/92552.105. Zugegriffen: 10.05.2021

Wagner, E., \& Schönau, D. (2016). Europäischer Referenzrahmen Visual Literacy. European Framework of Reference for Visual Literacy. Münster: Waxmann. 